\title{
UTILIZACIÓN DE AGROINSUMOS EN Paspalum atratum CV. CAMBÁ FCA
}

\author{
Agro-inputs usage in Paspalum atratum cv. Cambá FCA
}

\author{
Krynski, Mariel A. ${ }^{1}$; Romero, Amalia M. ${ }^{1}$; Urbani, Mario H. ${ }^{2}$ \\ ${ }^{1}$ Cátedra de Microbiología Agrícola, Facultad de Ciencias Agrarias, UNNE, Corrientes, Argentina. \\ ${ }^{2}$ Cátedra de Forrajicultura, Facultad de Ciencias Agrarias, UNNE; Corrientes, Argentina. \\ *E-mail: marielkrynski90@gmail.com
}

\begin{abstract}
RESUMEN
El objetivo del trabajo fue evaluar el efecto del uso de agroinsumos sobre la producción de biomasa de Paspalum atratum cv. Cambá y en la actividad biológica del suelo. El ensayo se realizó en macetas conteniendo suelo arenoso. Se aplicaron siete tratamientos con cinco repeticiones cada uno en un diseño completo al azar. Los tratamientos aplicados fueron: Testigo (T): suelo solo, Fertilizante Mineral (FM) $100 \mathrm{~kg} \cdot \mathrm{ha}^{-1}$, Lombricompost (LC) $40 \mathrm{tn} \cdot \mathrm{ha}^{-1}$, Lombricompost + Basalto (LC + B) 40 tn.ha $\mathrm{a}^{-1}$ y $2,5 \mathrm{tn}^{-h^{-1}}$ respectivamente, Compost (C) 40 tn.ha $a^{-1}$, Compost + Basalto (C+B) 40 tn.ha ${ }^{-1}$ y 2,5 tn.h.h ${ }^{-1}$ respectivamente y polvo de basalto (B) 2,5 tn.ha ${ }^{-1}$. Se realizaron 3 cortes, y en el último se extrajeron las plantas. Se midió altura, número de macollos, peso seco de parte aérea, peso seco radical y actividad biológica global. Se realizó ANAVA y comparación de medias por Tukey $(\alpha<0,05)$ observándose que para altura previo al primer corte B arrojó el menor valor, posterior al primer corte ningún tratamiento tuvo diferencias significativas. Para número de macollos no se encontraron diferencias significativas entre tratamientos hasta el primer corte, en las siguientes mediciones el tratamiento LC obtuvo los mayores valores y B los menores. Respecto a Peso Seco Aéreo en el primer corte no hubo diferencias significativas entre tratamientos y en los siguientes cortes el mayor valor lo tuvo el tratamiento LC. Para Peso Seco Radical el mayor valor lo obtuvo el tratamiento FM diferenciándose estadísticamente solo de B. Para Actividad Biológica Global no se encontraron diferencias estadísticamente significativas entre tratamientos.
\end{abstract}

Palabras clave: compost, Paspalum atratum, polvo de basalto, Actividad biológica.

\begin{abstract}
The aim of this work was to assess the effect of agro-inputs in biomass production of Paspalum atratum cv. Cambá and in soil biological activity. The sowing of pasture was carried out in pots with sandy soil. Seven treatments and five repetitions were applied; Control (T), Mineral Fertilizer (FM) 100 kg.ha ${ }^{1}$, Vermicompost (LC) 40 tn.ha ${ }^{-1}$, Vermicompost + Basalt $(\mathrm{LC}+\mathrm{B}) 40 \mathrm{tn}^{-h^{-1}}$ and 2.5 tn.ha $^{-1}$ respectively, Compost (C) 40 tn.ha ${ }^{-1}$, Compost + Basalt $(C+B) 40$ tn.ha ${ }^{-1}$ and 2.5 tn.ha ${ }^{-1}$ respectively and basalt powder (B) $2.5 \mathrm{tn} \cdot \mathrm{ha}^{-1}$. The plants were cut three times after that they were extracted. Height, amount of tillers, dry weight of the aerial part of the plants, radical dry weight and global biological activity were measured. ANOVA and means comparison by Tukey $(\alpha<0.05)$ were performed. When the first cut was carried out, the variable height obtained was the lowest in B treatment and after that measurement no other treatment denoted meaningful differences. The amount of tillers showed no meaningful differences among treatments until the first cut was performed. In the following measurements the LC treatment obtained the highest values and B the lowest ones. Regarding biomass aerial weight in the first cut, there were no meaningful differences between treatments and in the following cuts the LC treatment reached the highest value. On the other hand, the highest value of root dry weight was obtained by FM treatment, differing statistically only from B. The Global Biological Activity was similar among treatments.
\end{abstract}

Key words: compost, Paspalum atratum, Basalt powder, Biological activity.

Recibido: 24/jun/2020. Aceptado: 15/jul/2020 
Krynski, M.A. et al.: Utilización de agroinsumos en Paspalum atratum cv. Cambá.

\section{INTRODUCCIÓN}

Los pastizales y pasturas del noreste argentino (NEA) son las principales fuentes de alimentación para la producción de carne bovina. En su mayoría se tratan de especies estivales cuya característica principal es la producción desuniforme de forraje a lo largo de todo el año. Estos pastizales y pasturas muestran un patrón de crecimiento con 5 meses de alta producción de forraje (Noviembre a Marzo), 4 meses con producciones medias (Abril-Mayo y Septiembre-Octubre) y finalmente 3 meses de producciones muy bajas (Junio a Agosto) (Gándara et al., 2012).

Si bien las pasturas naturales son la base de la alimentación del ganado, las praderas implantadas de especies subtropicales son un recurso importante dentro de los planteos ganaderos del norte de la Argentina (Melgar et al., 2002). como en la provincia de Corrientes, y son herramientas de consideración cuando el objetivo es mejorar la producción y productividad de la empresa. Las pasturas y verdeos son una herramienta importante para la ganadería correntina, pueden ser utilizadas para intensificar la cría y diversificar la producción animal, siendo una práctica que puede ser implementada con éxito en diferentes áreas de la provincia, cuando se planifica el establecimiento y manejo (Altuve et al., 2003). Uno de los problemas que año tras año se debe afrontar es el déficit forrajero invernal, el cual puede superarse con el uso de verdeos invernales o mediante el diferimiento de la producción estival por medio de silaje, pasto diferido y rollos o fardos de heno. Un pasto diferido es una forma de transferir forraje del período de buena producción al período de escasez de alimento. La decisión de diferir en el tiempo el uso de una gramínea tropical, se asocia a la transferencia de cantidad de materia seca (MS), ya que la calidad de un diferido es baja (Ricci, 2003).

Entre las pasturas con buena aptitud y que entregan buena cantidad y calidad de MS que se pueden utilizar a la hora de planificar alguna estrategia para suplir la necesidad de forraje en el bache invernal se encuentra Paspalum atratum cultivar Cambá FCA. Este cultivar se logró por selección de poblaciones naturales de $P$. atratum, que fueron introducidas por la Facultad de Ciencias Agrarias en el año 1990 (Urbani et al., 1996). Esta variedad tiene la característica de producir una muy buena cantidad de semillas y de ser de reproducción apomíctica, por lo tanto, está garantizada la estabilidad genotípica (Quarín, et al. 1997). Es un cultivar perenne con desarrollo vegetativo principalmente en verano y que ha comenzado a sembrarse en las áreas Albardón del Paraná y Afloramientos Rocosos (Altuve et al., 2003). Produce forraje desde septiembre a mayo, no tiene plagas ni enfermedades importantes (Urbani et al., 1996). Presenta establecimiento por semillas, buen vigor de las plántulas, muy competitiva, adaptación a suelos ácidos y de baja fertilidad, buena producción de masa verde, buena aceptabilidad por los animales y alta digestibilidad de MS, tolera el pastoreo directo (Kalmbacher et al., 1997; Kalmbacher et al., 1995), es tardía para encañar (a fines de abril) y tiene su pico de producción a fines del verano. Además, es muy buena productora de semillas y se resiembra con facilidad. Si bien es un pasto algo duro cuando envejecen las hojas mantiene su alta palatabilidad; tiene muy buena respuesta al aumento de las temperaturas pasando el invierno y rebrota fácilmente (Saucedo et al., 2016). La producción de MS de forraje anual puede variar entre 3,7 y 36 tn.año ${ }^{-1}$ dependiendo de la frecuencia de defoliación, del clima, del suelo y de la fertilización aportada (Kardek, 2002).

La fertilización de los pastos, principalmente la nitrogenada, está entre los factores más importantes para determinar el nivel de producción por unidad de área (Primavesi et al., 2001). En la provincia de Corrientes es notoria la falta de fósforo en los suelos, debido a que el material original era deficiente en ese elemento. A este hecho se suman las características de ser suelos con $\mathrm{pH}$ ácidos $(4,5-5,5)$ y altos niveles de hierro y aluminio, que limitan la disponibilidad $\mathrm{P}_{2} \mathrm{O}_{5}$ para la planta a valores entre 1 y 5 ppm $\mathrm{P}_{2} \mathrm{O}_{5}$. Por todo lo antes mencionado, es que el agregado de fósforo a la siembra es una práctica necesaria para poder realizar cualquier pastura en sitios con esas características. En la implantación de una pastura pasa a ser fundamental la aplicación de fósforo dado que si no se realiza es frecuente que las malezas y el pastizal natural tengan ventaja de crecimiento a las plántulas de la pastura que está emergiendo, por estar más adaptados a la deficiencia de los suelos de la zona (Borrajo, 2007).

Una mejor nutrición del recurso forrajero permitirá aumentar su productividad, mejorar la calidad, aumentar la persistencia de la pradera y optimizar el período de aprovechamiento (Melgar et al., 2002). Una buena alternativa para suplir estas deficiencias de minerales lo constituyen los abonos orgánicos que se han usado desde tiempos remotos y su influencia sobre la fertilidad de los suelos se ha demostrado, aunque su composi- 
ción química, el aporte de nutrimentos a los cultivos y su efecto en el suelo varían según su procedencia, edad, manejo y contenido de humedad (Romero et al., 2000). Además, el valor de la materia orgánica que contiene, ofrece grandes ventajas que difícilmente pueden lograrse con los fertilizantes inorgánicos. Los abonos orgánicos (estiércoles, compostas y residuos de cosecha) aumentan la capacidad de retención de humedad en suelo y facilitan la disponibilidad de nutrientes para las plantas (López-Martínez et al., 2001). Entre las acciones para proteger los ecosistemas agropecuarios y prevenir su degradación, la aplicación de abonos orgánicos tiene una importancia significativa, dado que son el sostén básico para la vida en este medio y puede definir el potencial productivo del mismo (Paneque et al., 2004). Algunos de esos abonos orgánicos con buen aporte de nutrimentos lo constituyen las excretas de origen animal que poseen cantidades significativas de macro-nutrientes vegetales como Nitrógeno (N), Fósforo (P), Potasio (K), Calcio (Ca), Magnesio (Mg) y Azufre (S); y cantidades menores de micro-nutrientes como Zinc $(\mathrm{Zn})$ y Cobre $(\mathrm{Cu})$. Estos abonos aparte de mejorar las propiedades del suelo, a la vez también reducen la erosión y los costos afrontados por los productores en la compra de fertilizantes comerciales (Bogaard et al., 2013). El estiércol de caballo es muy interesante como fuente de materia orgánica para la agricultura y en especial para el compostaje y el vermicompostaje, debido a que es un material que se composta muy bien; el contenido en $\mathrm{N}$ es moderado con respecto a otros estiércoles como la "gallinaza" o el estiércol de oveja. A diferencia de otros animales como vacas u ovejas, los caballos no son rumiantes, por lo que su estiércol es ligeramente diferente (Tortosa, 2015).

Por su parte el lombricompostaje constituye una variación en la tecnología del compostaje, es un proceso biotecnológico que permite degradar y estabilizar residuos orgánicos bajo condiciones aeróbicas, mediante la acción de ciertas especies de lombrices capaces de alimentarse del residuo después de un proceso de pre compostaje, por la acción de sus enzimas digestivas y de la microflora aeróbica y anaeróbica presente en el interior del intestino de la lombriz. Entre los efectos favorables del lombricompostaje pueden citarse que produce un aumento general de la concentración de nutrientes, así como también favorece a que compuestos de la materia orgánica se mineralicen hasta formas químicas solubles que son fácilmente absorbibles por las plantas (Frioni, 2006).

En la producción agropecuaria, en otros países como Brasil está difundido el uso de la roca basáltica, por la gran posibilidad de suplir de nutrientes al suelo debido a su composición, especialmente $\mathrm{P}, \mathrm{Ca}, \mathrm{Mg}, \mathrm{y}$ micronutrientes; y por presentar en su mayoría bajo contenido de silicio (Ramos Gindri et al., 2014). Cuanto más finos son los polvos de esta roca, más rápida es su acción entre los microorganismos, característica importante debido a que no es un material soluble y hace falta un mínimo de actividad microbiana en el suelo para la disponibilidad de los nutrientes. En los suelos más pobres en vida microbiana se emplea el basalto muy finamente molido y en mayor cantidad, a cambio sus oligoelementos estimularan notablemente esa vida microbiana (Florin, 2006). La liberación de los nutrientes contenidos en la roca se realiza gracias a los ácidos orgánicos producidos por las plantas y microorganismos, así como por la biomasa en descomposición que se encuentra en el suelo.

El objetivo de este trabajo fue evaluar el efecto de agroinsumos sobre la producción de biomasa total de Paspalum atratum cv. Cambá y en la actividad biológica del suelo.

\section{MATERIALES Y MÉTODOS}

\section{Sustrato y agroinsumos utilizados.}

El suelo utilizado en el ensayo fue uno de textura arenosa obtenido de la Escuela Regional de Agricultura, Ganadería e Industrias Afines (ERAGIA), correspondiente a la serie Ensenada Grande. Esta serie se caracteriza por presentar escurrimiento medio, permeabilidad moderada, son bien drenados. En la superficie presenta un horizonte ócrico, espeso, de textura arenosa-franco y de reacción neutra; le sigue un horizonte BA arenosofranco, con presencia de lamelas texturales, débilmente estructurales, de color pardo oscuro y débilmente ácido. Son profundos, de buenas condiciones físicas para el desarrollo radical y penetración de agua. Son suelos de baja fertilidad, baja capacidad de retención de agua, con niveles de P moderados, con un contenido de MO menor al 1\% (Escobar et al., 1996). Los datos del análisis químico previo al ensayo se muestran en la Tabla 1.

El compost y lombricompost empleados fueron elaborados en la Cátedra de Microbiología Agrícola de la Facultad de Ciencias Agrarias (UNNE). El compost fue realizado a partir de estiércol equino; y el lombricom- 
Krynski, M.A. et al.: Utilización de agroinsumos en Paspalum atratum cv. Cambá.

puesto a partir de residuos orgánicos domiciliarios varios. La dosis aplicada en el ensayo tanto para lombricompost como para compost fue de 40 tn.ha ${ }^{-1}$.

El polvo de basalto es un residuo de la extracción y el procesamiento de la roca de basalto de una cantera ubicada en Curuzú Cuatiá (Ruta Nro. $119 \mathrm{~km} \mathrm{43300).} \mathrm{El} \mathrm{polvo} \mathrm{de} \mathrm{basalto} \mathrm{recolectado} \mathrm{fue} \mathrm{pasado} \mathrm{por} \mathrm{un} \mathrm{tamiz}$ de $0,5 \mathrm{~mm}$. La dosis utilizada fue de $2,5 \mathrm{tn} \cdot \mathrm{ha}^{-1}$.

El fertilizante mineral utilizado fue Hydrocomplex en su presentación granulada siendo éste altamente soluble. Aporta macro y micronutrientes, nitrógeno en forma de nitrato y amonio, fósforo en forma de polifosfatos y potasio a base de sulfato de potasio. Se aplicó una dosis equivalente a $100 \mathrm{~kg}_{\mathrm{ha}}{ }^{-1}$.

Tabla 1. Resultados obtenidos en el análisis químico de suelo, para las variables: $\mathrm{pH}, \mathrm{CE}$ (conductividad eléctrica) P (fósforo), $\mathrm{K}$ (potasio), Ca (calcio), Mg (magnesio), CO (carbono orgánico) y NT (Nitrógeno Total). Análisis realizado en el instituto Agrotécnico "Pedro M. Fuentes Godo" de la ciudad de Resistencia-Chaco.

\begin{tabular}{cccccccc}
\hline $\mathbf{p H}$ & $\begin{array}{c}\mathbf{C E} \\
\left(\mathbf{d S} . \mathbf{m}^{-1}\right)\end{array}$ & $\begin{array}{c}\mathbf{P} \\
\left(\mathbf{m g . k g}^{-1}\right)\end{array}$ & $\begin{array}{c}\mathbf{K} \\
\left(\mathbf{C m o l c ~ k g}^{-1}\right)\end{array}$ & $\begin{array}{c}\mathbf{C a} \\
\left(\mathbf{C m o l . k g}^{-1}\right)\end{array}$ & $\begin{array}{c}\mathbf{M g} \\
\left(\mathbf{C m o l c . k g}^{-1}\right)\end{array}$ & $\begin{array}{c}\mathbf{C O} \\
(\mathbf{\%})\end{array}$ & $\begin{array}{c}\mathbf{N T} \\
(\%)\end{array}$ \\
\hline 6.1 & 0.07 & 28.5 & 0.1 & 4.7 & 1.1 & 0.78 & 0.07 \\
\hline
\end{tabular}

\section{Ensayo realizado.}

Para el ensayo se utilizaron macetas de 5 litros de capacidad. Se incorporaron a los suelos de las macetas correspondientes las dosis de Fertilizante Mineral, Lombricompost, Lombricompost + Basalto, Compost, Compost + Basalto, y Basalto. Seguido a la incorporación de los agroinsumos se procedió a realizar la siembra colocando 10 semillas por maceta, las cuales fueron distribuidas al azar dentro de un invernáculo. En el ensayo se planteó un diseño completo al azar donde se aplicaron 7 tratamientos con 5 repeticiones con un total de 35 Unidades Experimentales (UE) (Tabla 2).

Tabla 2. Descripción de los tratamientos y las dosis aplicadas en el ensayo.

\begin{tabular}{|c|c|c|}
\hline Tratamientos & Descripción & Dosis \\
\hline $\mathrm{T}$ & Testigo (Suelo Solo) & - \\
\hline FM & Fertilizante Mineral & $0,1785 \mathrm{~g} / 5 \mathrm{~kg}$ de suelo. Equivalente a $100 \mathrm{~kg} \cdot \mathrm{ha}^{-1}$ \\
\hline $\mathrm{LC}$ & Lombricompost & $71 \mathrm{~g} / 5 \mathrm{~kg}$ de suelo. Equivalente a $40 \mathrm{tn} \cdot \mathrm{ha}^{-1}$ \\
\hline $\mathrm{LC}+\mathrm{B}$ & Lombricompost + Basalto & $\begin{array}{l}71 \mathrm{~g} / 5 \mathrm{~kg} \text { de suelo equivalente a } 40 \mathrm{tn} \cdot \mathrm{ha}^{-1}+4,45 \mathrm{~g} / 5 \\
\mathrm{~kg} \text { de suelo equivalente a } 2,5 \mathrm{tn} \cdot \mathrm{ha}^{-1} \text {, respectivamente }\end{array}$ \\
\hline $\mathrm{C}$ & Compost & $71 \mathrm{~g} / 5 \mathrm{~kg}$ de suelo equivalente a $40 \mathrm{tn} \cdot \mathrm{ha}^{-1}$ \\
\hline $\mathrm{C}+\mathrm{B}$ & Compost + Basalto & $\begin{array}{l}71 \mathrm{~g} / 5 \mathrm{~kg} \text { de suelo equivalente a } 40 \mathrm{tn} \cdot \mathrm{ha}^{-1}+4,45 \mathrm{~g} / 5 \\
\mathrm{~kg} \text { de suelo equivalente a } 2,5 \mathrm{tn} \cdot \mathrm{ha}^{-1} \text {, respectivamente }\end{array}$ \\
\hline B & Basalto & $4,45 \mathrm{~g} / 5 \mathrm{~kg}$ de suelo equivalente a $2,5 \mathrm{tn} \cdot \mathrm{ha}^{-1}$ \\
\hline
\end{tabular}

La emergencia de plántulas se inició a los 5 días posteriores a la siembra; se realizó riego y monitoreo de plagas y enfermedades durante todo el ensayo.

A los 30 días de la siembra se llevó a cabo un raleo de las plantas por maceta quedando 3 en cada una. Desde ese día se inició con las mediciones de altura, número de hojas y macollos, obteniendo así datos a los 30, 37 y 44 días, momento en el cual se realizó el primer corte de la pastura a la altura del puño mediante el uso de una tijera, tratando de esta manera simular el pastoreo de animales. Se continuó con las mediciones del rebrote para las variables de altura y número de macollos, a los 51, 58 y 62 días, punto en el cual se efectuó el segundo corte con los mismos criterios utilizados en el primer corte. A partir de ese momento solo se realizaron mediciones de altura debido a que el número de macollos se mantuvo constante, obteniendo datos a los 69,76 y 83 días, momento del último corte y extracción total del cultivo. 
Los cortes se realizaron con un espaciamiento entre ellos de aproximadamente 20 días, tomando un rango de porcentaje de cobertura entre $70-80 \%$.

En todos los cortes la parte aérea fue acondicionada para su secado en estufa. Con los tres cortes realizados se simuló un pastoreo. Los cortes se realizaron porque esta pastura requiere de cortes frecuentes para mantener la calidad y además también con la intención poder visualizar el efecto de los tratamientos sobre la capacidad de rebrote, mediante la obtención de peso seco en cada muestreo.

Para la extracción de la parte radical, se procedió a extraer las raíces cuidadosamente, separando el suelo adherido con golpes suaves. Una vez separadas las raíces, fueron lavadas y llevadas a estufa hasta peso contante para determinar de esta manera peso seco radical.

El suelo obtenido de cada tratamiento fue acondicionado, secado y tamizado para los análisis químicos correspondientes y de actividad biológica global, utilizando para los mismos tres repeticiones de cada tratamiento elegidos al azar.

\section{Actividad biológica global}

Se tomaron $30 \mathrm{~g}$ de suelo de cada tratamiento con tres (3) repeticiones, se procedió a humedecerlos a capacidad de campo y colocarlos en bolsitas. Las cuales fueron colocadas en frascos que contenían $40 \mathrm{ml}$ de hidróxido de sodio 0,5 Normal, también se prepararon tres blancos (frascos sin bolsitas conteniendo suelo), se llevaron a incubación a $28^{\circ} \mathrm{C}$ por siete días. Al cabo de la semana se procedió a la titulación con ácido Clorhídrico 0,5 Normal, y posteriormente se obtuvo la cantidad de $\mathrm{mg}$ de $\mathrm{CO}_{2}$ liberado mediante el uso de una fórmula (Frioni, 2011).

\section{Preparación y acondicionamiento de muestras para análisis foliar}

De las mismas repeticiones utilizadas para análisis químico de suelo y actividad biológica global, se tomaron muestras de las plantas obtenidas con el tercer corte. Las hojas fueron molidas por medio de un molinillo, fueron colocadas en sobres de papel debidamente rotulados, y enviados a laboratorio para realizar el análisis del contenido de nitrógeno y fósforo.

\section{Análisis estadístico}

Los resultados obtenidos fueron ordenados y tabulados para luego efectuar análisis de varianza y una comparación de medias entre tratamientos a través del test de Tukey con un $\alpha \leq 0,05$.

\section{RESULTADOS}

Los resultados obtenidos para el análisis de suelo al finalizar el ensayo son mostrados en la Tabla 3. Se puede observar en el contenido de nitrógeno entre los tratamientos, que si bien los abonos orgánicos en combinación con basalto se posicionaron primeros con los mayores valores, no hubo diferencias significativas. Las cantidades de nitrógeno obtenidas no variaron en gran medida respecto al contenido inicial del suelo (Tabla 1).

El contenido de fósforo fue mayor en el tratamiento Compost ( $\mathrm{P}>0,05)$, seguido de los tratamientos Lombricompost y Compost ambos en combinación con basalto. El tratamiento Compost alcanzó a diferenciarse de Lombricompost, Basalto, Testigo y Fertilizante Mineral; el tratamiento Lombricompost con basalto se diferenció de estos dos últimos y Compost con basalto no se diferenció estadísticamente con ningún tratamiento. Tanto basalto como fertilizante mineral fueron los de menor valor. Al comparar con los resultados del análisis de suelo pre ensayo (Tabla 1), se puede notar que los tratamientos que se ubicaron en primer lugar en la Tabla 3, es decir Compost solo, Lombricompost y Compost combinados con Basalto son los que tienen un contenido de Fósforo por encima del que originalmente tenía el suelo, el resto de los tratamientos disminuyó su contenido original de Fósforo en suelo.

En cuanto a la variable Potasio se puede observar que el mayor valor lo tuvo el tratamiento Compost y el menor valor el tratamiento Basalto, aunque no se encontraron diferencias estadísticamente significativas entre ninguno de los tratamientos; si comparamos con el análisis químico de suelo inicial, se ve que todos los tratamientos incrementaron su contenido de Potasio en suelo.

El aporte de basalto en forma única no obtuvo resultado en cuanto a los nutrientes analizados, en todos fue 
Krynski, M.A. et al.: Utilización de agroinsumos en Paspalum atratum cv. Cambá.

el de menor valor, al contrario de lo sucedido cuando su aporte es combinado con materiales orgánicos, donde en algunos casos fue el de mayor valor. Esto puede deberse a la dependencia que tiene este material de la actividad microbiana para su solubilidad (Lopes Motzuki et al., 2014; Camargo et al., 2012; Hinsinger et al., 2001)

En la Tabla 3 se muestran también los valores medios obtenidos en el análisis foliar, puede notarse que el contenido de nitrógeno foliar no fue significativamente diferente entre tratamientos. Sin embargo, los valores medios de fósforo mostraron diferencias significativas siendo el de mayor valor el tratamiento Compost en combinación con Basalto, diferenciándose de Testigo, Fertilizante Mineral, Lombricompost y Basalto. Al igual que el contenido de nitrógeno, el aporte de basalto resultó el de menor contenido foliar de fósforo. Los tratamientos con mayor contenido en fósforo fueron los mismos que tuvieron mayor contenido de fósforo en suelo, esto nos indica como el uso de abonos orgánicos además de aportar nutrientes al vegetal mantiene o hasta incluso mejora la fertilidad del suelo respecto al fósforo.

Tabla 3. Medias obtenidas a partir del análisis químico de las muestras de suelo al finalizar el ensayo (NTS: Nitrógeno Total en Suelo; PS: Fosforo en suelo; KS: Potasio en Suelo) y Valores medios de contenido foliar para nitrógeno $(\mathrm{NF})$ y fósforo $(\mathrm{PF})$. Medias con una letra común no son significativamente diferentes $(\mathrm{p}>$ $0.05)$.

\begin{tabular}{|c|c|c|c|c|c|}
\hline Tratamientos & NTS (\%) & PS (mg.kg $\left.{ }^{-1}\right)$ & KS (Cmolc. $\left.\mathrm{kg}^{-1}\right)$ & NF (\%) & PF (mg.kg-) \\
\hline Testigo & $0.05 \mathrm{~A}$ & $19.10_{\mathrm{BC}}$ & $0.05 \mathrm{~A}$ & $1.00 \mathrm{~A}$ & $0.30_{\mathrm{C}}$ \\
\hline $\begin{array}{l}\text { Fertilizante } \\
\text { Mineral }\end{array}$ & $0.05 \mathrm{~A}$ & $14.93 \mathrm{C}$ & $0.05 \mathrm{~A}$ & $0.82 \mathrm{~A}$ & $0.33_{\mathrm{BC}}$ \\
\hline Lombricompost & $0.06_{\mathrm{A}}$ & $24.83 \mathrm{BC}$ & $0.05 \mathrm{~A}$ & $1.00 \mathrm{~A}$ & $0.33 \mathrm{BC}$ \\
\hline $\begin{array}{l}\text { Lombricompost } \\
+ \text { Basalto }\end{array}$ & $0.07 \mathrm{~A}$ & $34.93 \mathrm{AB}$ & $0.05 \mathrm{~A}$ & $1.00_{\mathrm{A}}$ & $0.40_{\mathrm{ABC}}$ \\
\hline Compost & $0.07 \mathrm{~A}$ & $44.83_{\mathrm{A}}$ & $0.06_{\mathrm{A}}$ & $0.79 \mathrm{~A}$ & $0.43 \mathrm{AB}$ \\
\hline $\begin{array}{l}\text { Compost } \\
+ \text { Basalto }\end{array}$ & $0.07 \mathrm{~A}$ & $31.57 \mathrm{ABC}$ & $0.05 \mathrm{~A}$ & $0.89 \mathrm{~A}$ & $0.50_{\mathrm{A}}$ \\
\hline \multirow[t]{2}{*}{ Basalto } & $0.06_{\mathrm{A}}$ & $14.10_{\mathrm{C}}$ & $0.04_{\mathrm{A}}$ & $0.70_{\mathrm{A}}$ & $0.30_{\mathrm{C}}$ \\
\hline & $\mathrm{CV}: 21.31$ & $C V: 25.28$ & $C V: 10.49$ & $C V: 45.51$ & $C V: 10.18$ \\
\hline
\end{tabular}

En la Tabla 4 se muestran los datos obtenidos para la variable Número de Macollos en las mediciones realizadas. Puede observarse que a los 44 días post siembra (momento del $1^{\mathrm{er}}$ corte) ningún tratamiento se diferenció estadísticamente de otro. Para la siguiente medición, se pudieron apreciar diferencias significativas entre el tratamiento Lombricompost y el tratamiento Basalto obteniendo este último un menor valor; manteniéndose esta tendencia en la medición posterior. Contrariamente a los 62 días post siembra (momento correspondiente al $2^{\text {do }}$ corte) ya no se encontraron diferencias significativas entre tratamientos.

Tabla 4. Resultados obtenidos para la variable Número de Macollos. Medias con una letra común no son significativamente diferentes $(\mathrm{p}>0.05)$.

\begin{tabular}{|c|c|c|c|c|c|c|}
\hline Tratamientos & $\begin{array}{l}30 \text { días post } \\
\text { siembra }\end{array}$ & $\begin{array}{l}37 \text { días post } \\
\text { siembra }\end{array}$ & $\begin{array}{c}4 \text { días post } \\
\text { siembra } \\
\left(1^{\mathrm{er}} \text { corte }\right)\end{array}$ & $\begin{array}{l}51 \text { días post } \\
\text { siembra }\end{array}$ & $\begin{array}{l}58 \text { días post } \\
\text { siembra }\end{array}$ & $\begin{array}{c}2 \text { días post } \\
\text { siembra } \\
\left(2^{\text {do }} \text { corte }\right)\end{array}$ \\
\hline Testigo & $2.00_{\mathrm{A}}$ & $3.27 \mathrm{~A}$ & $6.40_{\mathrm{A}}$ & $6.47 \mathrm{AB}$ & $6.47 \mathrm{AB}$ & $6.47 \mathrm{~A}$ \\
\hline $\begin{array}{l}\text { Fertilizante } \\
\text { Mineral }\end{array}$ & $3.13 \mathrm{~A}$ & $4.80_{\mathrm{A}}$ & $6.27 \mathrm{~A}$ & $6.40 \mathrm{AB}$ & $6.53 \mathrm{AB}$ & $6.40_{\mathrm{A}}$ \\
\hline Lombricompost & $3.07 \mathrm{~A}$ & $4.33_{\mathrm{A}}$ & $7.13_{\mathrm{A}}$ & $7.67 \mathrm{~A}$ & $7.87 \mathrm{~A}$ & $7.53_{\mathrm{A}}$ \\
\hline $\begin{array}{l}\text { Lombricompost } \\
+ \text { Basalto }\end{array}$ & $3.20_{\mathrm{A}}$ & $4.87 \mathrm{~A}$ & $6.80_{\mathrm{A}}$ & $6.93 \mathrm{AB}$ & $6.93 \mathrm{AB}$ & $6.93 \mathrm{~A}$ \\
\hline Compost & $2.87 \mathrm{~A}$ & $3.87 \mathrm{~A}$ & $5.93 \mathrm{~A}$ & $6.07 \mathrm{AB}$ & $6.27 \mathrm{AB}$ & $6.27 \mathrm{~A}$ \\
\hline $\begin{array}{l}\text { Compost } \\
+ \text { Basalto }\end{array}$ & $3.00 \mathrm{~A}$ & $4.53 \mathrm{~A}$ & $6.93 \mathrm{~A}$ & $7.00 \mathrm{AB}$ & $7.13 \mathrm{AB}$ & $6.87 \mathrm{~A}$ \\
\hline \multirow[t]{2}{*}{ Basalto } & $2.13_{\mathrm{A}}$ & $3.27 \mathrm{~A}$ & $4.20_{\mathrm{A}}$ & $4.20_{\mathrm{B}}$ & $4.27 \mathrm{~B}$ & $4.40_{\mathrm{A}}$ \\
\hline & CV: 66.86 & CV: 56.09 & CV: 46.90 & CV: 48.23 & $C V: 49.22$ & CV: 49.64 \\
\hline
\end{tabular}


Los datos obtenidos para la variable altura se muestran en la Tabla 5 (datos correspondientes a los momentos de primer, segundo y tercer corte). Se puede observar que a los 44 días post siembra (momento del $1^{\mathrm{er}}$ corte) se encontraron diferencias significativas solamente entre el tratamiento testigo con el mayor valor frente al tratamiento Basalto con el menor valor. En las mediciones posteriores no se encontraron diferencias estadísticamente significativas entre ninguno de los tratamientos.

En cuanto a la variable peso seco de parte aérea, datos mostrados también en la tabla 5, a los 44 días post siembra $\left(1^{\mathrm{er}}\right.$ corte) no se encontraron diferencias significativas entre los tratamientos. A los 62 días post siembra ( $2^{\text {do }}$ corte) el mayor valor lo obtuvo el tratamiento Lombricompost diferenciándose estadísticamente de los tratamientos Testigo, Fertilizante Mineral y Basalto siendo este último el del menor valor. En la medición final, a los 83 días post siembra ( $3^{\mathrm{er}}$ corte), el tratamiento Lombricompost mantuvo diferencias con Fertilizante Mineral y Basalto.

Tabla 5. Resultados obtenidos para las variables Altura $(\mathrm{H})$ y Peso Seco de parte Aérea (PSA) Medias con una letra común no son significativamente diferentes $(\mathrm{p}>0.05)$.

\begin{tabular}{|c|c|c|c|c|c|c|}
\hline \multirow[t]{2}{*}{ Tratamientos } & \multicolumn{2}{|c|}{$\begin{array}{c}44 \text { días post siembra( } 1^{\mathrm{er}} \\
\text { corte) }\end{array}$} & \multicolumn{2}{|c|}{$\begin{array}{c}62 \text { días post siembra }\left(2^{\mathrm{d} 0}\right. \\
\text { corte })\end{array}$} & \multicolumn{2}{|c|}{$\begin{array}{c}83 \text { días post siembra ( } 3^{\text {er }} \\
\text { corte })\end{array}$} \\
\hline & $\mathrm{H}(\mathrm{cm})$ & PSA (g) & $\mathrm{H}(\mathrm{cm})$ & PSA (g) & $\mathrm{H}(\mathrm{cm})$ & $\operatorname{PSA}(\mathrm{g})$ \\
\hline Testigo & $62.07 \mathrm{~A}$ & $2.34_{\mathrm{A}}$ & $52.65 \mathrm{~A}$ & $1.89 \mathrm{BCD}$ & $46.49 \mathrm{~A}$ & $2.28 \mathrm{ABC}$ \\
\hline $\begin{array}{l}\text { Fertilizante } \\
\text { Mineral }\end{array}$ & $60.90_{\mathrm{AB}}$ & $2.60 \mathrm{~A}$ & $51.02 \mathrm{~A}$ & $1.72 \mathrm{CD}$ & $45.17 \mathrm{~A}$ & $2.09 \mathrm{BC}$ \\
\hline Lombricompost & $57.35 \mathrm{AB}$ & $2.62_{\mathrm{A}}$ & $55.47 \mathrm{~A}$ & $2.97 \mathrm{~A}$ & $46.16_{\mathrm{A}}$ & $3.18_{\mathrm{A}}$ \\
\hline $\begin{array}{l}\text { Lombricompost } \\
+ \text { Basalto }\end{array}$ & $60.31_{\mathrm{AB}}$ & $3.13 \mathrm{~A}$ & $51.71_{\mathrm{A}}$ & $2.49 \mathrm{ABC}$ & $46.41_{\mathrm{A}}$ & $2.78 \mathrm{AB}$ \\
\hline Compost & $61.97 \mathrm{AB}$ & $2.71_{\mathrm{A}}$ & $54.87 \mathrm{~A}$ & $2.24 \mathrm{ABCD}$ & $48.76_{\mathrm{A}}$ & $2.40_{\mathrm{ABC}}$ \\
\hline $\begin{array}{l}\text { Compost } \\
+ \text { Basalto }\end{array}$ & $61.58 \mathrm{AB}$ & $2.83 \mathrm{~A}$ & $56.43 \mathrm{~A}$ & $2.71 \mathrm{AB}$ & $47.65 \mathrm{~A}$ & $2.77 \mathrm{AB}$ \\
\hline \multirow[t]{2}{*}{ Basalto } & 55.99 в & $1.99 \mathrm{~A}$ & $51.03 \mathrm{~A}$ & $1.42 \mathrm{D}$ & $45.48 \mathrm{~A}$ & $1.74_{\mathrm{C}}$ \\
\hline & CV: 9.10 & CV: 49.51 & CV: 10.29 & CV: 38.34 & CV:9.75 & CV: 36.19 \\
\hline
\end{tabular}

Con respecto a la variable Peso seco radical (Tabla 6), el mayor valor lo obtuvo el tratamiento Fertilizante Mineral con diferencias significativas frente al tratamiento Basalto, siendo este último el que menor valor arrojó.

Los resultados obtenidos en la medición de actividad biológica global se muestran también en la Tabla 6, en la cual puede observarse que los valores no mostraron diferencias estadísticamente significativas entre tratamientos.

Tabla 6. Resultados obtenidos para las variables Peso Seco Radical (PSR) y Actividad Biológica Global $(\mathrm{ABG})$. Medias con una letra común no son significativamente diferentes $(\mathrm{p}>0.05)$.

\begin{tabular}{lcc}
\hline \multicolumn{1}{c}{ Tratamientos } & PSR $(\mathbf{g})$ & ABG $\left(\mathbf{m g} \mathbf{C O}_{\mathbf{2}} / \mathbf{1 0 0}\right.$ g de suelo) \\
\hline Testigo & $14.90_{\mathrm{AB}}$ & $31.53_{\mathrm{A}}$ \\
\hline Fertilizante Mineral & $15.94_{\mathrm{A}}$ & $35.93_{\mathrm{A}}$ \\
\hline Lombricompost & $15.09_{\mathrm{AB}}$ & $35.93_{\mathrm{A}}$ \\
\hline Lombricompost + Basalto & $14.12_{\mathrm{AB}}$ & $28.60_{\mathrm{A}}$ \\
\hline Compost & $10.51_{\mathrm{AB}}$ & $30.07_{\mathrm{A}}$ \\
\hline Compost + Basalto & $11.43_{\mathrm{AB}}$ & $21.27_{\mathrm{A}}$ \\
\hline Basalto & $7.30_{\mathrm{B}}$ & $30.07_{\mathrm{A}}$ \\
\hline & $\mathrm{CV}: 32.06$ & $\mathrm{CV}: 47.24$ \\
\hline
\end{tabular}


Krynski, M.A. et al.: Utilización de agroinsumos en Paspalum atratum cv. Cambá.

\section{CONSIDERACIONES FINALES}

En el cultivo de Paspalum atratum cv cambá el uso de lombricompost puede incrementar la producción en materia seca de la parte aérea y promover una mejor respuesta en macollaje o acumulación de materia seca post cortes o pastoreos de la pastura.

La adición de basalto a los suelos no tuvo efectos positivos sobre el cultivo para ninguna de las variables; sin embargo, al combinarlo con los tratamientos orgánicos la respuesta de las variables medidas se mostró favorable.

La actividad biológica global en los suelos analizados no se vio influenciada por ninguno de los tratamientos.

El tratamiento fertilizante mineral promovió el incremento del peso seco radical de la pastura pero tuvo un comportamiento muy diverso en cuanto a las otras variables medidas.

\section{BIBLIOGRAFÍA}

Altuve, S. y Bendersky, D. (2003). Pasturas y verdeos en Corrientes, establecimiento y producción. E.E.A. INTA Mercedes. Sitio Argentino de Producción Animal. Noticias y Comentarios No 379. http:// www.produccionanimal.com.ar/produccion_y_manejo_pasturas/pasturas_cultivadas_megatermicas/27 -pasturas_y_verdeos_en_corrientes.pdf

Bogaard, A., Fraser, R., Heaton Wallace, M., Vaiglova, P., Charles, M., Jones, G., Evershed, R., Styring, A., Andersen, N., Arbogast, R., Bartosiewicz, L., Gardeisen, A., Kanstrup, M., Maier, U., Marinova, E., Ninov, L., Schäfer, M. y Stephan, E. (2013). Crop manuring and intensive land management by Europe's first farmers, Proceedings of the National Academy of Sciences 110 (31): 1258912594.

Borrajo, C. (2007). Pasturas subtropicales en el NEA. Curso internacional en ganadería bovina subtropical. Modulo II implantación de pasturas subtropicales. Sitio Argentino de Producción Animal. http:// www.produccion-animal.com.ar/produccion_y_manejo_pasturas/

pasturas_cultivadas_megatermicas/78-borrajo.pdf

Camargo, K., Vilela de Resende, J., Pinheiro Camargo, L., Figueredo, A. y Zanin, D. (2012). Produtividade do morangueiro em função da adubação orgânica e com pó de basalto no plantio. Semina: Ciências Agrárias, Londrina, Vol. 33 (1): 2985-2994.

Escobar, E., Ligier, H., Melgar, R., Matteio, H. y Vallejos, O. (1996). Mapa de suelos de la provincia de Corrientes. E.E.A., INTA. Corrientes, Argentina. Disponible en: http:// www.geointa.inta.gob.ar/2016/09/23/suelos-de-la-provincia-de-corrientes-1500-000/

Florin, X. (2006). El basalto en la agricultura. Revista la fertilidad de la tierra 24. 49-52. http:// www.mapama.gob.es/ministerio/pags/Biblioteca/Revistas/pdf_Ferti/Ferti_2006_24_48_52.pdf

Frioni, L. (2011). Microbiología: básica, ambiental y agrícola. $1^{\mathrm{a}}$. ed. Orientación Gráfica Editora. Buenos Aires. 786 p.

Frioni, L. (2006). Microbiología básica, ambiental y agrícola. Facultad de Agronomía. Universidad de la República, Uruguay. $463 \mathrm{pp}$.

Gándara, L., Pereira, M.M. y Slukwa, M. (2012). AER INTA Corrientes. Alternativas tecnológicas para incrementar la producción forrajera. Sitio argentino de producción animal. http:// www.produccionbovina.com.ar/produccion_y_manejo_pasturas/ pasturas_cultivadas_megatermicas/190-INCREMENTAR.pdf

Hinsinger, P., Fernandes Barros, O., Benedetti, M., Noack, Y. y Callot, G. (2001). Plant-induced weathering of a basaltic rock: experimental evidence. Geochimica et Cosmochimica Acta, Vol. 65 (1): 137-152,https://doi.org/10.1016/S0016-7037(00)00524-X

Kalmbacher, R., Brown, W.,Colvin, D., Dunavin L., Kretschmer, J., Martin, F., Mullahey, J. y Rechcigl, J. (1997). Suerte Atra Paspalum. its management and utilization. University of Florida - Agricultural Experiment Station - Circular S-397cirs-397. https://rcrec-ona.ifas.ufl.edu/media/rcrec-onaifasufledu/ pdf/publications/suerte-atra-paspalum.pdf 
Kalmbacher, R. y Kretschmer, A. (1995) Suerte Atra Paspalum. In: INTERNATIONAL CONFERENCE ON LIVESTOCK IN TROPICS, Gainesville. Proceedings Florida: University of Florida, 1995. p. 13 21

Kardek, A. (2002) Avaliações agronômicas de genótipos de Paspalum spp no âmbito dos cerrados. 288 f. Tese (Doutorado) Universidade Estadual de São Paulo, Jaboticabal. https://ainfo.cnptia.embrapa.br/digital/ bitstream/CPAC-2009/21342/1/ramos_01.pdf

López-Martinez, J., Díaz, A., Martínez, E. y Valdez, R. (2001). Abonos orgánicos y su efecto en propiedades físicas y químicas del suelo y rendimiento en maíz. Terra Latinoamericana, Vol. 19 (4): 293-299. http://www.redalyc.org/html/573/57319401/

Lopes Motizuki, O., Carrillho, E., Lopes Assad, M. (2014). Effect of rock powder and vinasse on two types of soils. R. Bras. Ci. Solo, 38:1547-1557.

Melgar, R. y Torres, D. (2002). Forrajeras subtropicales. Sitio argentino de producción animal.

Paneque, V. y Calaña, J. (2004). Manual abonos orgánicos. Conceptos prácticos para su evaluación y aplicación. Instituto Nacional de Ciencias Agrícolas. La Habana, Cuba. 37 p.

Primavesi, O., Primavesi, A., Correa, A. y Silva, C. (2001) Composição bromatológica de coastcross submetido a diferentes doses de nitrogênio. In: REUNIÃO ANUAL DA SOCIEDADE BRASILEIRA DE ZOOTECNIA, 38.

Quarín, C.,Valls, J. y Urbani, M. (1997). Cytological and reproductive behavior of Paspalum atratum, a promising forage grass for the tropics. Tropical Grasslands, 31, 114-116.

Ramos Gindri C., Gislaine de Mello, A. y Kautzman Müller, R. (2014). A preliminary study of acid volvanic rocks for stonemeal application. Environmental Nanotechnology Monitoring and management. 1-2: 30-35.

Ricci, H. (2003). Diferido: preparándonos para el invierno. Revista de la Sociedad Rural de Jesús María 138: 28-30.

Romero Lima, M.R, Trinidad Santos A., García Espinosa, R. y Ferrara Cerrato, R. (2000). Producción de papa y biomasa microbiana en suelo con abonos orgánicos y minerales. Agrociencia 34 (3): 261-269. https://www.redalyc.org/pdf/302/30234302.pdf

Saucedo, M., Castro, C. y Obregón, H. (2016). Revista Voces y Ecos № 35: 47-49.

Tortosa, G. (2015). Materiales para compostar: estiércol de caballo. Compostando ciencia. http:// www.compostandociencia.com/2015/02/materiales-para-compostar-estiercol-de-caballo/

Urbani, M., Quarín, C., Espinoza, F. y Norrmann, G. (1996). Inscripción en el Registro de la Propiedad de Cultivares del Pasto "Cambá FCA"; una Forrajera para el Nordeste. Actas de la Reunión de Comunicaciones Científicas y Tecnológicas. UNNE, Tomo III p. 81-84. 